\title{
Partial restoration of blink reflex function after spinal accessory-facial nerve anastomosis
}

\author{
Nicolas Danziger, Bénédicte Chassande, Georges Lamas, Isabelle Fligny, \\ Jacques Soudant, Jean Claude Willer
}

\begin{abstract}
Functional motor control requires perfect matching of the central connections of motoneurons with their peripheral inputs. It is not known, however, to what extent these central circuits are influenced by target muscles, either during development or after a lesion. Surgical interventions aimed at restoring function after peripheral nerve lesions provide an opportunity for studying this interaction in the mature human nervous system. A patient was studied in whom the spinal accessory nerve was anastomosed into a lesioned facial nerve, allowing voluntary contractions of the previously paralysed muscles. This procedure, in addition to replacing the facial neurons at peripheral synapses, allowed a new short latency trigeminospinal accessory reflex of the $\mathrm{R} 1$ blink reflex type to be demonstrated, implying that trigeminal neurons had sprouted towards spinal accessory motoneurons over a distance of at least $1 \mathrm{~cm}$. These results show an unexpected influence of the periphery in remodelling central connectivity in humans. The motoneuronal excitability for this R1 reflex response was therefore studied to compare the convergent properties of facial motoneurons (normal side) with those of the spinal accessory motoneurons (operated side) using a classic double shock technique with variable interstimulus intervals (conditioning test stimulus). On the normal side, conditioning stimuli (to the ipsilateral or contralateral infraliminar supraorbital nerve) produced a clearcut facilitation of the $R 1$ blink reflex when the interstimulus interval was $30-80 \mathrm{~ms}$. By contrast, a similar procedure had no effect on the R1 blink reflex mediated via the trigeminalspinal accessory reflex arc. These data indicate that despite the heterotopic sprouting of some axons from neurons in the trigeminal principal nucleus towards the XIth nucleus, those motoneurons involved in the newly formed reflex arc remain totally inexcitable by other trigeminal afferents and seem unable to ensure a physiological functioning of the normal blink reflex. Thus the functional relevance of the recovered R1 blink response remains unclear.
\end{abstract}

$(\mathcal{F}$ Neurol Neurosurg Psychiatry 1995;58:222-226)
Keywords: spinal accessory-facial nerve anastomosis; synaptic reorganisation; neuronal plasticity; blink reflex pathways

In normal subjects, electrical stimulation of the supraorbital nerve elicits a well known two component blink reflex response in the orbicularis oculi muscles: the first one (R1) has a short latency (around $10 \mathrm{~ms}$ ), and is strictly ipsilateral to the stimulus, whereas the second component (R2) has a longer latency (around $30-35 \mathrm{~ms}$ ), acts bilaterally, and is responsible for the clinical closure of the eyelid. ${ }^{12}$ The R1 response follows high rates of stimulation (up to $1 \mathrm{~Hz}$ ) without being modified and the R2 component habituates at a stimulation rate of $0.25 \mathrm{~Hz}^{2-7}$ The $\mathrm{R} 1$ response is mediated in the periphery by fast conducting $\mathrm{A} \beta$ fibres $^{348}$; in mammals, its central path is disynaptic and completely intrapontine. ${ }^{9-12}$ After a relay in the trigeminal principal (main sensory) nucleus, the afferent signals are carried directly to the ipsilateral intermediate facial subnucleus, which contains motoneurons innervating the orbicularis oculi muscles. ${ }^{13-15}$ The $R 2$ response is known to be mediated by peripheral afferents similar to those for the $\mathrm{R} 1$ response. ${ }^{23}$ Its central circuitry is largely polysynaptic and has been extensively described elsewhere. ${ }^{9-11}$

We have shown recently that in patients in whom the hypoglossal nerve (XIIth cranial nerve) was anastomosed into an axotomised lesioned facial nerve (VIIth cranial nerve), this resulted not only in a replacement of facial motoneurons at peripheral synapses, but also of a new short latency trigeminohypoglossal reflex of the $\mathrm{R} 1$ blink reflex type, which could be demonstrated without an R2 component. ${ }^{19}$ This surprising finding was explained in terms of the sprouting of axons from trigeminal neurons towards hypoglossal motoneurons, thus making new trigeminohypoglossal connections that allowed the restoration of a function which existed before the peripheral lesion.

We now report the finding that in a patient with a spinal accessory-facial nerve (XI-VII) crossover, a synaptic reorganisation of the $\mathrm{R} 1$ component of the blink reflex through an unusual pathway occurs, showing the functional importance of the blink reflex response in the protective reactions of the eyeball.

\section{Methods}

After agreement of a local committee and informed consent, we studied the electrophysiological features of the trigeminofacial blink 
reflex (a brainstem reflex) in three normal subjects (two men, one woman, aged 38 to 50 years) and in a patient (a 52 year old man) who had undergone a peripheral spinal accessory-facial nerve (XI-VII) anastomosis as a part of his treatment after removal of a section of the facial nerve. This patient had had an extensive cerebellopontine neuroma of the left acoustic nerve which impinged on the facial nerve, necessitating the removal of a large part of the nerve during surgical ablation of the neuroma (in 1971). The restorative XI-VII crossover had been carried out about one month after the surgical ablation of the neuroma. In the interval, the patient had clinical signs of a total infranuclear facial palsy, showing that the functioning of the motor axons of the VIIth nerve was totally impaired. All reflex contractions were abolished. About 13 to 15 months after the surgical anastomosis, the patient had clinical signs of partial recovery of facial muscle activity, to the extent that he could perform some specific voluntary contractions such as blinking, sucking, or making faces. Importantly, laryngeal motor function was normal, showing that the cranial root of the XIth nerve was not involved in the graft.

The present study was undertaken in November 1993 - that is, more than 20 years after the restorative surgery. At that time, direct motor responses, recorded with standard EMG techniques, were noted in the facial muscles after electrical stimulation of the peripheral branches of the anastomosis in its preauricular pathway. This showed that the peripheral reinnervation of the facial muscles by the spinal accessory motor axons was functionally active. Table 1 gives the values for the motor responses from facial muscles on the normal and operated sides. Also, specific voluntary contractions of the eyelids associated with the classic recruitment of motor units in the facial muscles were recorded. Synkinesiae were also seen in these muscles after voluntary movements of either the neck or the shoulder thus showing that motoneurons of the XIth but not of the VIIth nerve were involved in the process of reinnervation.

The electrophysiological procedure for studying the blink reflex has been described extensively elsewhere. ${ }^{126}$ Briefly, the subjects were comfortably installed reclining in a bed with a head rest, to ensure a state of general muscle relaxation. The blink reflex was evoked by electrical stimulation of the supraorbital nerve with a pair of surface electrodes. Square wave single pulses $(0.1 \mathrm{~ms})$ were delivered at a rate of $0 \cdot 16 \mathrm{~Hz}$ by a constant current stimulator. Reflex responses were recorded on EMG from the orbicularis oculi muscles (pars inferior) on both sides through surface electrodes.

We also explored the excitability of the R1 reflex to compare the ability of facial (normal side) and accessory spinal (operated side) motoneurons to fire in response to a conditioning stimulus. In other words, the convergent properties of each motoneuronal pool were studied to investigate to what extent the V-XI R1 blink reflex response could functionally replace the normal V-VII R1 blink reflex response. For this purpose, we used a classic double shock technique. ${ }^{57}$ Briefly, the test stimulus (ipsilateral supraorbital nerve) was adjusted to elicit stable blink reflex responses while the conditioning stimulus (either ipsilateral or contralateral supraorbital nerve) was adjusted to just subthreshold for any reflex response. The $\mathrm{R} 1$ reflex response was integrated and measured within a $15 \mathrm{~ms}$ time window starting $8 \mathrm{~ms}$ after the test stimulus onset. In these conditions, after a five minute control period when only the test stimulus was delivered, both stimuli were applied together with an interstimulus interval $(\Delta t)$ of between 0 and $100 \mathrm{~ms}$. As previous studies had shown that the use of random or increasing time intervals yielded similar data, ${ }^{7}$ we used the increasing time interval on the normal and on the operated side. Signals on EMG were full wave rectified, integrated, and analysed on line with a computerised system. Numerical values for the $\mathrm{R} 1$ response were then converted to percentages to allow intraindividual comparisons between unconditioned and conditioned responses (the $100 \%$ value represented the mean of 40 responses obtained during the control period preceding each conditioning procedure).

\section{Results}

ELECTROPHYSIOLOGICAL FEATURES OF THE BLINK REFLEX RESPONSE

In normal subjects and on the patient's normal (right) side, electrical stimulation of the supraorbital nerve elicited the classic, two component, blink reflex responses in the orbicularis oculi muscles: the ipsilateral R1 had a short latency $(10.2$ (SD $0.4 \mathrm{~ms})$ and the bilateral R2 a longer latency (31 (SD $2.3 \mathrm{~ms}$ ); fig $1 \mathrm{~A})$. The $\mathrm{R} 1$ response followed high rates of stimulation (up to $1 \mathrm{~Hz}$ ) without being modified whereas the $\mathrm{R} 2$ component habituated at a stimulation rate of $0.25 \mathrm{~Hz}$. Furthermore, these responses were also modulated by central influences as evidenced by increases in

Electrophysiological characteristics of the direct motor responses elicited from facial muscles by facial nerve stimulation on the normal (right) side and by stimulation of the XI-VII crossover on the operated (left) side

\begin{tabular}{|c|c|c|c|c|c|c|}
\hline \multirow[b]{2}{*}{ Muscles } & \multicolumn{3}{|c|}{ Normal (right side) } & \multicolumn{3}{|c|}{ Operated (left side) } \\
\hline & Threshold $(m A)$ & Latency (ms) & Amplitude ( $m V$ ) & Threshold $(m A)$ & Latency (ms) & Amplitude ( $m V$ ) \\
\hline $\begin{array}{l}\text { Frontalis } \\
\text { Orbicularis oculi } \\
\text { Orbicularis labii } \\
\text { Chin }\end{array}$ & $\begin{array}{l}2 \cdot 1 \\
1 \cdot 9 \\
2 \cdot 2 \\
2 \cdot 1\end{array}$ & $\begin{array}{l}3 \cdot 1 \\
2 \cdot 9 \\
3 \cdot 3 \\
3 \cdot 3\end{array}$ & $\begin{array}{l}4 \cdot 2 \\
3 \cdot 4 \\
4 \cdot 1 \\
5 \cdot 3\end{array}$ & $\begin{array}{l}3 \cdot 2 \\
2 \cdot 2 \\
3 \cdot 5 \\
3 \cdot 1\end{array}$ & $\begin{array}{l}4 \cdot 2 \\
3 \cdot 8 \\
3 \cdot 5 \\
4 \cdot 4\end{array}$ & $\begin{array}{l}3 \cdot 9 \\
3 \cdot 3 \\
3 \cdot 5 \\
3 \cdot 6\end{array}$ \\
\hline
\end{tabular}

Values obtained on the normal side are similar to those from normal subjects whereas on the operated side there are small increases in the thresholds and latencies and a small decrease in the amplitude of the motor responses. 
Figure 1 Examples of blink reflex responses

elicited by stimulation of each supraorbital nerve, recorded from the

orbicularis oculi muscles on the normal $(A)$ and operated $(B)$ sides. Right supraorbital nerve stimulation (normal side) elicits a typical two component blink reflex response with normal latencies in the ipsilateral muscle $(R 1=10.3 \mathrm{~ms}$;

$R 2=33 \mathrm{~ms}$ ) whereas no response is recorded in the contralateral muscle. By contrast, supraorbital nerve stimulation applied to the left (operated) side elicits an unexpected $R 1$ reflex response at a latency of $12.5 \mathrm{~ms}$ in the ipsilateral muscle. Such a stimulus also elicits a normal, crossed, R2 blinking response ( 34 ms latency) in the contralateral orbicularis oculi muscle. Each trace shows the responses to six successive stimuli superimposed.

Figure 2 Recordings of the $R 1$ blink reflex response from the left orbicularis oculi muscle (operated side) without (control) and with conditioning stimulation of the contralateral supraorbital nerve at various interstimulus intervals (from 20 to $100 \mathrm{~ms}$ ). Note the lack of any effect of the conditioning stimuli on the $R 1$ test response. Each trace shows four successive responses superimposed.

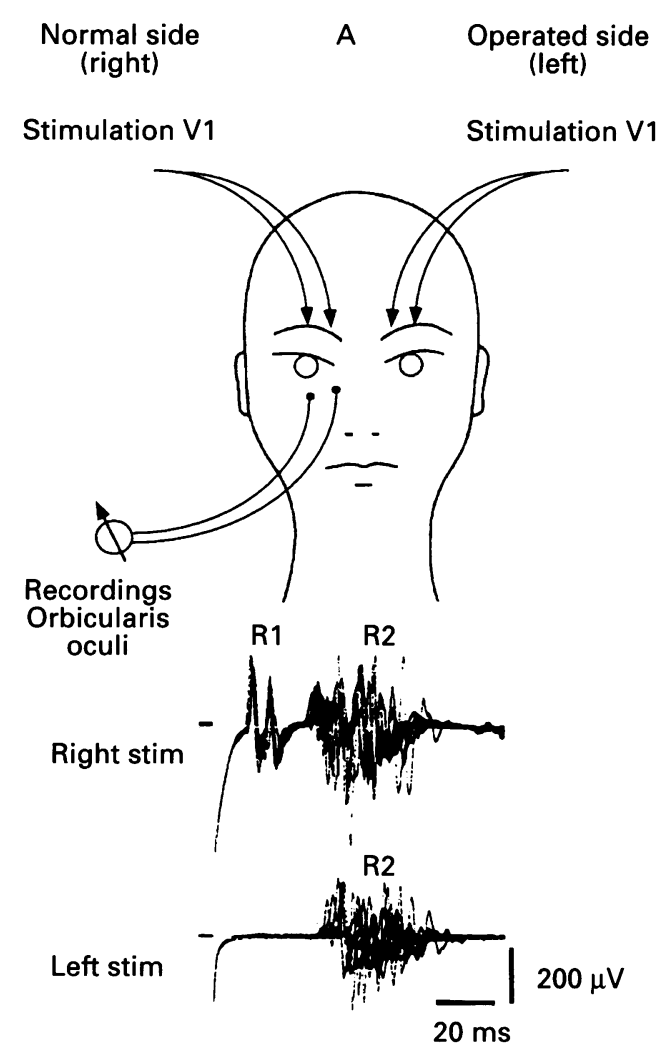

Normal side (right)

Stimulation V1 (left) side (left)

Left stim

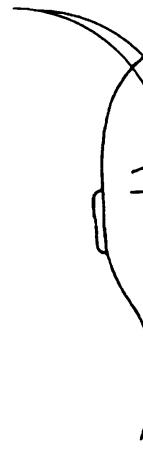

Stimulation V1

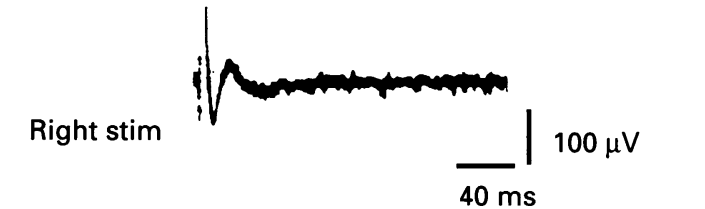

their amplitudes during small voluntary contractions of the eyelids.

On the patient's operated (left) side, supraorbital nerve stimulation elicited an unexpected reflex response of the R1 type $(12.5$ ms) that was not followed by an R2 component in the ipsilateral muscles (fig 1B). As with normal subjects and on the patient's normal side, this response did not show habituation or wind up facilitation at high rates of stimulation (up to $1 \mathrm{~Hz}$ ), showing that it was a genuine short latency and oligosynaptic reflex

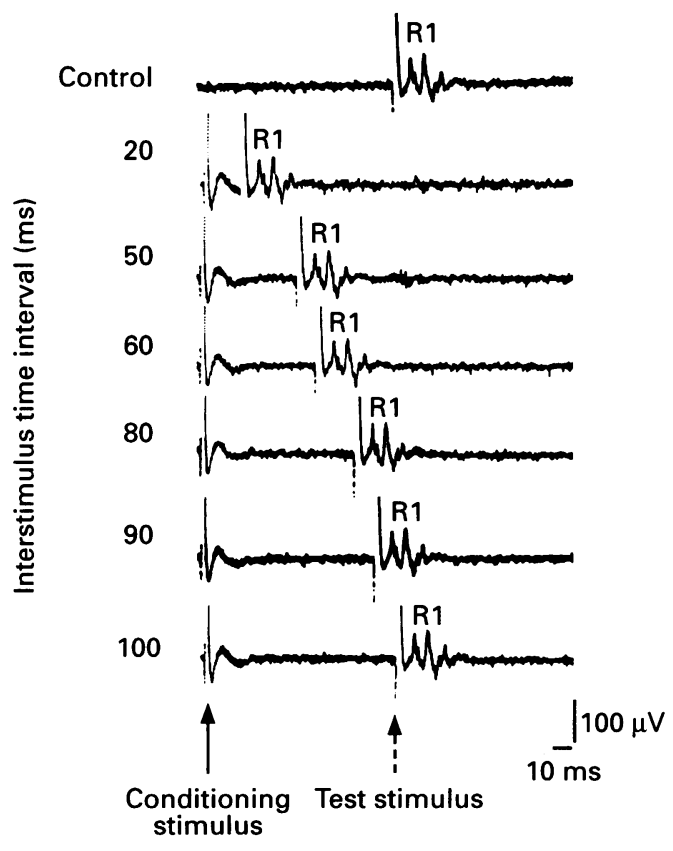

component of the R1 type and not part of a startle reaction, which in any case would have a longer latency (30-40 $\mathrm{ms}^{17}$ ). Interestingly and by contrast with the normal side, this response remained constant in amplitude $(0 \cdot 1$ $\mathrm{mV}$ ) throughout the investigations, behaving as if it was not modulated centrally, and this was also shown by the lack of any facilitatory effect of voluntary contractions of the eyelids on this side.

In a series of control experiments in normal subjects and on the patient's normal side, supraorbital nerve stimulation did not elicit any reflex activity in the sternocleidomastoid or trapezius muscles, even at high intensities (up to $30 \mathrm{~mA}$ ), which were clearly activating smaller diameter cutaneous afferents of the $\mathrm{A} \delta$ type in that they produced a pinprick type pain. Conversely, electrical stimulation (up to $15 \mathrm{~mA}$ ) of the spinal accessory nerve along its peripheral course did not produce any direct motor or reflex responses in the facial muscles.

\section{EXCITABILITY CYCLE OF THE R1 COMPONENT} OF THE BLINK REFLEX

On the patient's normal side, as in normal control subjects, conditioning stimuli applied ipsilaterally or contralaterally resulted in a clearcut facilitation of the $\mathrm{R} 1$ response, which became significant with an interstimulus interval $(\Delta \mathrm{t})$ of $30 \mathrm{~ms}(+50 \%)$, increased progressively to a maximum facilitatory effect ( + $200 \%$ ) with a $\Delta t$ of $50 \mathrm{~ms}$, and decreased progressively to recover baseline values with $\Delta t$ values of 80-90 ms.

By contrast, on the patient's operated side, neither ipsilateral nor contralateral conditioning stimuli produced any significant change 
Figure 3 Excitability cycle of the R1 blink reflex response on the right (normal) and left (operated) side. In both graphs, the conditioning stimuli were applied successively to ipsilateral and contralateral supraorbital nerves. On the normal side, the facilitatory effect of the conditioning stimulus began at an interstimulus interval of $30 \mathrm{~ms}$, reached its maximum at $50 \mathrm{~ms}$, and recovered towards 70-80 ms. By contrast, on the operated side these procedures showed no effect on the $R 1$ blink reflex response. On both graphs each value corresponds to the mean of 10 successive responses.

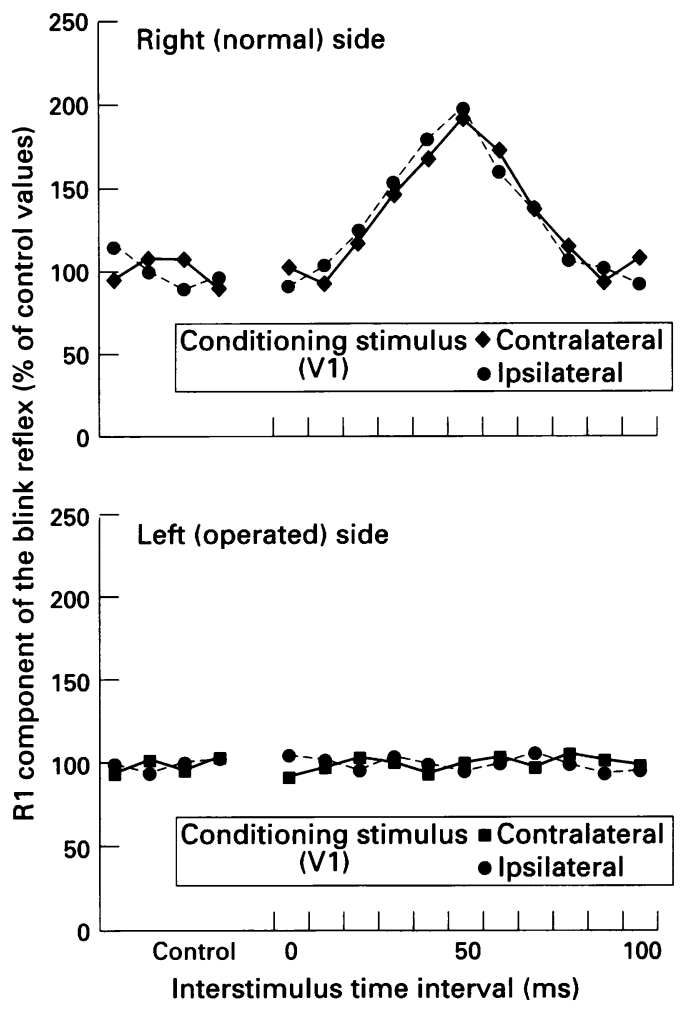

in the $\mathrm{R} 1$ trigeminospinal accessory reflex response (figs 2 and 3). Furthermore the R1 reflex response remained remarkably stable in shape and amplitude throughout the session (fig 2).

\section{Discussion}

As both the non-functional facial motor nucleus and facial motor axons were bypassed by the peripheral anastomosis, our present data strongly suggest that the short latency $R 1$ blink reflex response recorded in the orbicularis oculi muscles on the operated side was mediated centrally through an oligosynaptic and newly formed V-XI reflex arc. Like all other motor nuclei, the spinal nucleus of the XIth nerve receives direct and indirect afferent projections from various peripheral sources and these mediate reflex activities related to head and neck movements. Up to the present time, projections from the trigeminal principal sensory nucleus to the spinal nucleus of the XIth nerve have not been described in humans or in animals. As this structure is known to be a relay for the R1 blink reflex, it is tempting to suggest tentatively that the most probable explanation for the recovery of this response on the patient's operated side is the heterotopic sprouting of axons of trigeminal neurons from the principal trigeminal nucleus towards the nearest motoneurons of the spinal nucleus of the XIth nerve. Given the distance between the inferior pole of the facial nucleus and this structure, ( 1 to $1.5 \mathrm{~cm}$ in humans), ${ }^{18}$ this sprouting must be extensive. This hypothesis is supported by several arguments: (1) according to data from autoradiographic tracing studies in cats and rabbits, strong ipsilateral projections to motoneuronal cell groups involved in blinking have been found from the ventrolateral pontine tegmental field and from the medullary medial tegmental field at the levels of the hypoglossal nucleus and nucleus ambiguus, ${ }^{11} 19-22$ but not that of the spinal nucleus of the XIth nerve. Furthermore, it is clear that these projections are not involved in the pathway for the R1 blink reflex. ${ }^{1011}$ (2) In animals and in humans there are no anatomical or physiological data at present that suggest the existence of connections between cutaneous trigeminal afferents and the spinal root of the XIth nerve. Thus a hypothesis that the peripheral anastomosis discloses a pre-existing but silent circuit seems unlikely. (3) In normal human subjects, there is neither clinical nor electrophysiological evidence for a V-XI reflex as electrical stimulation of the supraorbital nerve does not elicit activity in the sternocleidomastoid or the trapezius muscles. (4) In normal humans, stimulation of the XIth nerve along its peripheral course does not elicit any direct motor or reflex response in the facial muscles even with strong electrical stimuli (up to $30 \mathrm{~mA}$ ).

In addition to this hypothesis of a surprising, albeit possible, new V-XI connection, there are alternative hypotheses to explain our data. These include a regrowth of facial nerve axons either from the central sectioned stump (near the geniculi ganglion) directly towards facial muscles, or from the facial motor nucleus through the lower part of the nucleus ambiguus towards facial muscles via the anastomosis. Although theoretically possible, these alternatives seem unlikely because: $(a)$ the facial nerve was cut first twice, once, near the brainstem during the operation for the neuroma, and then in the periphery to allow the anastomosis to be achieved; (b) the findings of synkinesiae in facial muscles during shoulder or neck movements clearly indicate that motoneurons of the spinal root of the XIth and the VIIth but not the facial nerve were involved in this process.

Thus our electrophysiological study has unexpectedly indicated that some central neuronal networks in the adult human CNS can be structurally modified as a function of their peripheral environment, allowing the restoration of a function in which they were involved before the occurrence of a peripheral lesion.

The study of the excitability cycle of the R1 component shows that on the normal side, as in normal subjects, the conditioning stimulation induced a facilitatory effect on the R1 reflex within latencies $(30 \mathrm{~ms}$ to $80 \mathrm{~ms}$ ) that correspond to the impinging of subthreshold R2 afferent signals on the facial motoneuronal pool regardless of the side to which the conditioning stimuli were applied. Thus it seems that in physiological conditions, the facilitation of the R1 component is due to the R2 afferent volley, which may increase the firing of facial motoneurons involved in the $R 1$ response. This suggests that various types of trigeminal afferent signals project and converge on to facial motoneurons to give the functional blink reflex response. By contrast, 
on the operated side, the lack of any facilitatory effect of either the ipsilateral or the contralateral conditioning stimulus can be explained easily by the lack of any $\mathrm{R} 2$ blink reflex response on this side even with strong ipsilateral or contralateral supraorbital nerve stimulation (up to $20 \mathrm{~mA}$ ). This result suggests that only those trigeminal afferent neurons responsible for the transmission of the R1 blink reflex are involved in sprouting towards spinal accessory motoneurons. The lack of any $\mathrm{R} 2$ reflex response mediated through the trigeminospinal accessory motoneuron arc is not surprising as it is difficult to think that a multisynaptic reorganisation associated with multiple, heterotopic, and extensive sprouting could occur in such a long arborised pathway. Finally, it seems that despite the heterotopic sprouting of some axons from the trigeminal principal nucleus towards the XIth nucleus, those motoneurons involved in the neoformed reflex arc seem to be deafferented as they remain totally unexcitable by conditioning trigeminal afferent signals or by other types of central modulation such as pyramidal tract facilitation. Thus they seem to be unable to ensure the physiological functioning of the normal blink reflex, which is a bilateral closure of the eyelids. Furthermore, as the R1 response also remained unmodified during voluntary contractions of the orbicularis oculi muscles, it can be assumed that those motoneurons involved in the R1 blink reflex pathway are different from those involved in the voluntary closure of the eyelids. Taken together, these findings raise important questions concerning the relative importance of central and peripheral mechanisms in the processes of synaptic plasticity in the adult human CNS that remodel, in a different and independent fashion, the voluntary and reflex inputs to motoneurons that are apparently involved in similar functions - that is, the motor functions of the face.

We thank JP Bardon and M Chastanet for their helpful technical assistance and Dr S Cadden for his valuable help with the English. This work was supported by CRC-AP-HP No 912209; 922202; CRE INSERM 920814; and by La Fondation pour La Recherche Médicale.

1 Kugelberg E. Facial reflexes. Brain 1952;75:385-96.

2 Ongerboer de Visser BW. Anatomical and functional organization of reflexes involving the trigeminal system in man: jaw reflex, blink reflex, corneal reflex and exteroceptive suppression. Adv Neurol 1983;39:727-38.

3 Cruccu G, Bowsher D. Intracranial stimulation of the trigeminal nerve in man. II: reflex responses. 7 Neurol Neurosurg Psychiatry 1986;49:419-27.

4 Cruccu G, Inghilleri M, Fraioli B, Guidetti B, Manfredi $M$. Neurophysiologic assessment of trigeminal function after surgery for trigeminal neuralgia. Neurology 1987; 37:631-8.

5 Trontelj MA, Trontelj JV. Reflex arc of the first component of the human blink reflex: a single motoneuron study. F Neurol Neurosurg Psychiatry 1978;41:538-47.

6 Willer JC, Lamour Y. Electrophysiological evidence for a facio-facial reflex in the facial muscles in man. Brain Res 1977;119:459-64.

7 Willer JC, Roby A, Boulu P, Boureau F. Comparative effects of electroacupuncture and transcutaneous nerve stimulation on the human blink reflex. Pain 1982;14: 267-78.

8 Lindquist C, Martensson A. Mechanisms involved in the cat's blink reflex. Acta Physiol Scand 1970;80:149-59.

9 Hiraoka $M$, Shimamura $M$. Neural mechanisms of the corneal blinking reflex in cats. Brain Res 1977;125: 265-75.

10 Holstege G. Neuronal organization of the blink reflex. In: Paxinos G, ed. The human nervous system. San Diego: Paxinos G, ed. The human nerod

11 Holstege G, Tan J, Van Ham JJ, Graveland GA. Anatomical observations on the afferent projections to the retractor bulbi motoneuronal cell group and other pathways possibly related to the blink reflex in the cat. Brain Res 1986;374:321-34.

12 Takeuchi $Y$, Nakano $K$, Uemura $M$, Matsuda $K$, Matsushima R, Mizuno N. Mesencephalic and pontine afferent fiber system to the facial nucleus in the cat: a study using the horseradish peroxidase and silver impregnation techniques. Exp Neurol 1979;66:330-42.

13 Holstege G, Kuypers HGJM, Dekker JJ. The organization of the bulbar fibre connections to the trigeminal, facial and hypoglossal motor nuclei. II. An autoradiographic tracing study in cat. Brain 1977; 100:265-86.

14 Holstege G, Tan J, Van Ham JJ, Bos A. Mesencephalic projections to the facial nucleus in the cat. An autoprojections to the facial nucleus in the cat. An auto-
radiographical tracing study. Brain Res 1984;311:7-22.

radiographical tracing study. Brain Res 1984;311:7-22.
15 Tamai $Y$, Iwamoto $M$, Tsujimoto $T$. Pathway of the blink reflex in the brainstem of the cat: interneurons between the trigeminal nuclei and the facial nucleus. Brain Res 1986;380:19-25.

16 Willer JC, Lamas G, Poignonec S, Figny I, Soudant J. Redirection of the hypoglossal nerve to facial muscles alters central connectivity in human brainstem. Brain Res 1992;594:301-6.

17 Shimamura M. Neural mechanisms of the startle reflex in cerebral palsy, with special reference to its relationships with spino-bulbo-spinal reflexes. In: Desmedt JE, ed. New developments in electromyography and clinical neurophysiology. Vol 3. Basel: Karger, 1973;761-6.

18 Carpenter MB, Sutin J. Human neuroanatomy. Baltimore: Williams and Wilkins, 1983:315-57.

19 Aldes LD, Boone TB. Organization of projections from the principal sensory trigeminal nucleus to the hypoglossal nucleus in the rat: an experimental light and electron microscopic study with axonal tracer techniques. Exp Brain Res 1985;59:16-29.

20 Harvey JA, Land T, McMaster SE. Anatomical study of the rabbit's corneal-VIth nerve reflex: connections between cornea, trigeminal sensory complex, and the abducens and accessory abducens nuclei. Brain Res 1984;301:307-21.

21 Amri M, Car A, Roman C. Axonal branching of medullary swallowing neurons projecting on the trigeminal and hypoglossal motor nuclei: demonstration by electrophysiological and fluorescent double labeling techniques. Exp Brain Res 1990;81:384-90.

22 Olsson KA, Westberg K-G. Interneurons in the trigeminal motor system. In: Van Steenberghe D, De Laat A, eds. EMG of jaw reflexes in man, Leuven: Leuven University Press, 1989:19-50. 